\title{
A network of superconducting gravimeters as a detector of matter with feeble nongravitational coupling ${ }^{\star}$
}

\author{
Wenxiang $\mathrm{Hu}^{1}$, Matthew M. Lawson ${ }^{2,3}$, Dmitry Budker ${ }^{4,5}$, Nataniel L. Figueroa ${ }^{4}$, a Derek F. Jackson Kimball ${ }^{6}$, \\ Allen P. Mills Jr. ${ }^{7}$, and Christian Voigt ${ }^{8}$ \\ 1 School of Physics, Peking University, Beijing, P.R. China \\ 2 The Oskar Klein Centre for Cosmoparticle Physics, Department of Physics, Stockholm University, AlbaNova, \\ Stockholm 10691, Sweden \\ 3 Nordita, KTH Royal Institute of Technology and Stockholm University, Roslagstullbacken 23, Stockholm 10691, Sweden \\ 4 Helmholtz Institut, Johannes Gutenberg-Universität Mainz, Mainz 55128, Germany \\ 5 Department of Physics, University of California, Berkeley, CA 94720, USA \\ 6 Department of Physics, California State University - East Bay, Hayward, CA, USA \\ 7 Department of Physics and Astronomy, University of California, Riverside, CA, USA \\ 8 GFZ German Research Centre for Geosciences, Telegrafenberg, Potsdam, Germany
}

Received 5 February 2020 / Received in final form 31 March 2020

Published online 11 June 2020

(C) The Author(s) 2020. This article is published with open access at Springerlink.com

\begin{abstract}
Hidden matter that interacts only gravitationally would oscillate at characteristic frequencies when trapped inside of Earth. For small oscillations near the center of the Earth, these frequencies are around $300 \mu \mathrm{Hz}$. Additionally, signatures at higher harmonics would appear because of the non-uniformity of Earth's density. In this work, we use data from a global network of gravimeters of the International Geodynamics and Earth Tide Service (IGETS) to look for these hypothetical trapped objects. We find no evidence for such objects with masses on the order of $10^{14} \mathrm{~kg}$ or greater with an oscillation amplitude of $0.1 r_{e}$. It may be possible to improve the sensitivity of the search by several orders of magnitude via better understanding of the terrestrial noise sources and more advanced data analysis.
\end{abstract}

\section{Introduction}

A classic result in Newtonian gravity is that if a small mass is orbiting inside a large mass of uniform density, such that the orbit is entirely contained in the interior of the large mass, the period of the orbit is fixed by the density of the large mass, and independent of the particulars of the orbit. This is because the system can be described as a threedimensional harmonic oscillator. In the case of a mass inside a sphere with uniform density equal to the average density of the Earth, the period of such orbits would be approximately $80 \mathrm{~min}$. Such a scenario is impossible with masses comprised of ordinary matter because of nongravitational interactions. However, the situation could be hypothetically realized if the small mass is comprised of some "hidden matter" (we call it a hidden internal object, HIO) that has only feeble, if any, non-gravitational interactions with normal matter. Furthermore, it is known that such hidden matter exists: evidence from many indepen-

\footnotetext{
* Contribution to the Topical Issue "Quantum Technologies for Gravitational Physics", edited by Tanja Mehlstäubler, Yanbei Chen, Guglielmo M. Tino and Hsien-Chi Yeh.

${ }^{a}$ e-mail: figueroa@uni-mainz.de
}

dent observations point to the existence of dark matter [1], an invisible substance which may interact with ordinary matter primarily via gravity. If some fraction of this hidden matter is gravitationally bound within the Earth, this hypothetical scenario of a hidden internal object could be realized.

This suggests a tantalizing scenario. Perhaps, one can detect the presence of such HIO via sensitive measurements of gravitational acceleration at the surface of the Earth. An attractive feature of this idea is that the method does not depend on any specifics of what the orbiting matter is composed of and, in the case of uniform density, would lead to a signal at a well defined frequency. Unfortunately, the latter condition does not hold for the case of the Earth: the density profile of the Earth [2] (Fig. 1) is far from being uniform. Nevertheless, there may be situations leading to distinct spectral features, for example, if the HIO undergoes small oscillations near the center of the Earth where the density is nearly uniform.

Sensitive gravimetry measurements are performed with a variety of instruments [3]; among the most sensitive ones is a global network of gravimeters, the International Geodynamics and Earth Tide Service (IGETS [4,5]) 


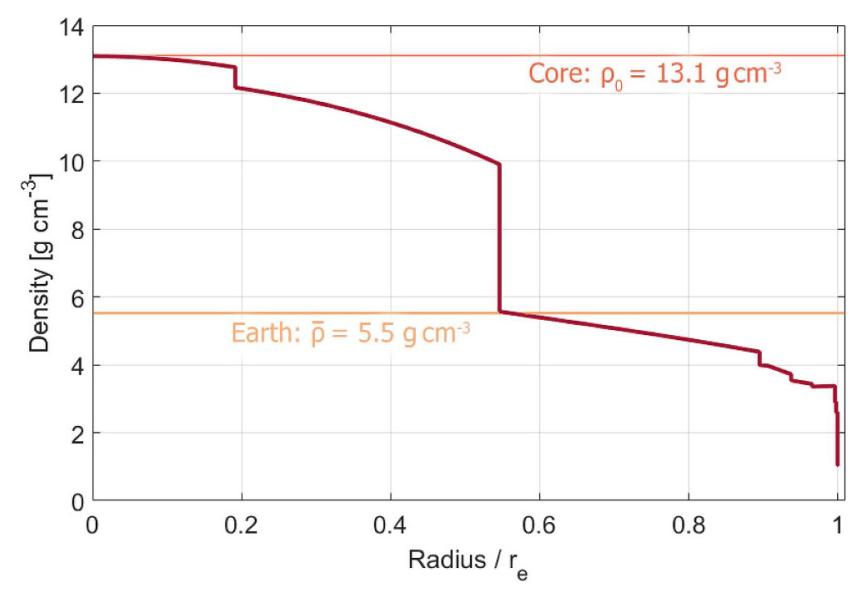

Fig. 1. The density profile of the Earth based on the Preliminary Reference Earth model (PREM) [2]. $r_{e}=6371 \mathrm{~km}$ is the mean radius of the Earth, $\bar{\rho}=5.51 \mathrm{~g} \mathrm{~cm}^{-3}$ is the average density of the Earth, and $\rho_{0}=13.1 \mathrm{~g} \mathrm{~cm}^{-3}$ is the density at the Earth's center.

discussed in more detail in Section 4. If HIOs exist inside the Earth, each gravimeter in the network would see a weak periodic signal at its characteristic frequencies, with a phase depending on the geometry of the orbit and location of the gravimeter. The presence of such frequency components in a Fourier analysis of the gravimeter timesequence data could indicate the presence of a HIO if it can be adequately differentiated from naturally occurring spectral features. This methodology is similar to that used by geophysicists to search for periodic oscillations of the solid core of the Earth, the so-called Slichter mode [6,7].

There are also entirely different scenarios that can lead to signals in principle observable with gravimeters. For example, ultralight scalar dark-matter field can lead to effective variation of fundamental constants, including the mass of the baryons [8]. This would cause a sinusoidal variation of the Earth mass at a frequency equal to the oscillation frequency of the dark-matter field. This could be, for example, the background galactic dark matter nominally oscillating at the Compton frequency of the underlying boson [8], an Earth-bound halo [9], or the field in a "boson star" encountering the Earth [10] and leading to a transient (rather than a persistent) signal. Some such scenarios have been recently analyzed in reference [11]. The data from the gravimeter network were also used in reference [12] to set limits on a possible violation of Lorentz invariance.

In this paper, we survey the scenarios that could potentially lead to observable effects of the HIO, discuss the sensitivity of a gravimeter network, and present a preliminary analysis of a historical record of the IGETS data. Finally, we assess the prospects of the future HIO searches based on these techniques.

During the preparation of this manuscript, we became aware of a similar work [13]. While the basic idea and approach of reference [13] are close to ours, the specifics are different and complementary.

\section{Capture/formation scenarios and their difficulties}

One can imagine a few different scenarios in which an oscillatory gravitational signal can be produced by hidden sector objects gravitationally bound to the Earth. The most studied in the literature (see e.g., $[9,14]$ ) is the formation of diffuse halos of dark matter particles around the Earth ${ }^{1}$. One could imagine a scenario in which a meteor impact or other violent jolt set up relative oscillations between such a halo and Earth. However, numerical models of a halo of non-interacting particles on orbits around a point mass have shown that any overall coherent oscillations of the halo would damp out on orbital time scales and become unobservable. This can be seen intuitively by noting that each particle in the halo has (generally) a different orbital frequency, thus the overall oscillations will not add coherently.

A second scenario is massive compact hidden-sector objects on orbits within, or possibly extending slightly beyond, the surface of the Earth. As previously noted (and as will be quantified below), objects with such trajectories do not have the enticing property of single orbitindependent orbital frequencies, and thus are in general difficult to detect by the methods discussed in this paper.

The third scenario is massive compact objects on orbits confined sufficiently close to the center of the Earth where the Earth's density is essentially uniform. It is such objects which we will principally consider. We thus define a Hidden Internal Object (HIO) as a compact object that orbits as a single object, entirely within the Earth's core.

How could HIOs be formed? In general this is a difficult problem for non/minimally interacting objects. An object starting far from the Earth following a trajectory that will bring it within the interior of the Earth starts with a gravitational potential energy equal to the necessary energy to reach the Earth's escape velocity. Moreover, the velocity of generic objects in the galaxy relative to the Earth is generally on the order of the Milky Way virial velocity of $220 \mathrm{~km} \mathrm{~s}^{-1}$, whereas the escape velocity for the Earth is $11 \mathrm{~km} \mathrm{~s}^{-1}$, so some strongly inelastic process is needed for even orbital capture, and additional energy dissipation is required to localize the object to the inside of the Earth. Objects of interest to us might be expected, in general, to have nearly dissipationless interactions with ordinary matter. However, this does not necessarily exclude dissipation due to self-interactions or interactions with other forms of dark matter in the hidden sector. For example, one possible capture scenario is that a captured object originates from a diffuse "cloud" in which a small part is sheared away and gets captured by the Earth in an

\footnotetext{
1 One model suggests that axion quark nuggets (AQN) [15] explain the similarity of the dark and visible cosmological matter densities: in this model annihilation of anti-AQNs with visible matter produces a terrestrial halo of axion dark matter when AQNs hit the Earth. Although only a small fraction $\left(\approx 10^{-17}\right)$ of the emitted particles stay bound, the accumulation of axions over the history of the Earth can still result in a halo (see [14] for a detailed discussions of the process), although in this scenario the halo is external, virial, and of order $0.1 \mathrm{~kg}$ and thus not suitable for detection with gravimeters.
} 
effective "three-body" collision. Such scenarios are not uncommon in celestial dynamics, where gravitational tidal forces can rip apart bound objects and capture material [16]. The self-interaction scenario, however, has two serious problems. One is that it requires the hidden-sector object to be only weakly self-bound so that some of the material can be gravitationally sheared off, but this type of weak self interaction will generically lead to either the formation of rings of the material (making our detection scheme unworkable) or to complete virialization (with the same effect). Additionally this scenario requires the matter being captured to have non-trivial self-interactions, which are generically constrained by galaxy cluster mergers [17].

A further difficulty is that even if a dense object is captured into an Earth orbit, only extremely eccentric orbits (those intersecting the Earth) plus a dissipative interaction between the compact object and the Earth offers a plausible method for confining the object to the interior of Earth. But, such an interaction would damp the orbit until the object is confined to be mostly stationary at the center of the Earth. A possible alternative energy-loss mechanism related to gravitational polarization of the Earth is discussed in Appendix B.

We also note that regardless of capture mechanism, any damping mechanism which is enhanced for large velocities and suppressed for small velocities will tend to produce circular orbits.

We emphasize that a specific consistent scenario for $\mathrm{HIO}$ formation is yet to be worked out.

To give a sense of scale to our discussions of HIO masses, we consider several quantities. The total amount of dark matter enclosed in a sphere with a radius equal to that of the solar system (under the assumptions of the standard halo model and assuming uniform density of the dark matter) is $\approx 3 \times 10^{17} \mathrm{~kg}$, while that contained in a sphere with the radius of the Earth is on the order of $1 \mathrm{~kg}$. Another interesting mass to compare is obtained by considering the volume $\mathcal{V}$ traced out through the galaxy by the Earth as it has travelled through space since its formation: $\mathcal{V}=v A T \approx 10^{36} \mathrm{~m}^{3}$, where $v \approx 2 \times 10^{5} \mathrm{~m} / \mathrm{s}$ is the speed of the Earth relative to the galactic rest frame, $A \approx 10^{14} \mathrm{~m}^{2}$ is the Earth's cross-sectional area, and $T \approx 4.5 \times 10^{9}$ years is the age of the Earth. Multiplying $\mathcal{V}$ by the average dark matter density $\rho_{\mathrm{DM}} \approx$ $0.4 \mathrm{GeV} / c^{2} / \mathrm{cm}^{3} \approx 7 \times 10^{-22} \mathrm{~kg} / \mathrm{m}^{3}$ (here $c$ is the speed of light), we get that the total dark matter mass the Earth has passed through is about: $\mathcal{M} \sim 10^{15} \mathrm{~kg}$. The feeble interactions between dark matter and baryonic matter makes this quantity of dark matter unachievable in normal capture scenarios but it can serve as an upper limit on dark matter capture.

\section{Detection signatures}

As mentioned above, the non-uniformity of the Earth's density leads to broadening in the spectrum of the HIO orbital frequencies, nominally removing the attractive feature of the original idea that one may just look for orbits at a single unique and predictable frequency.

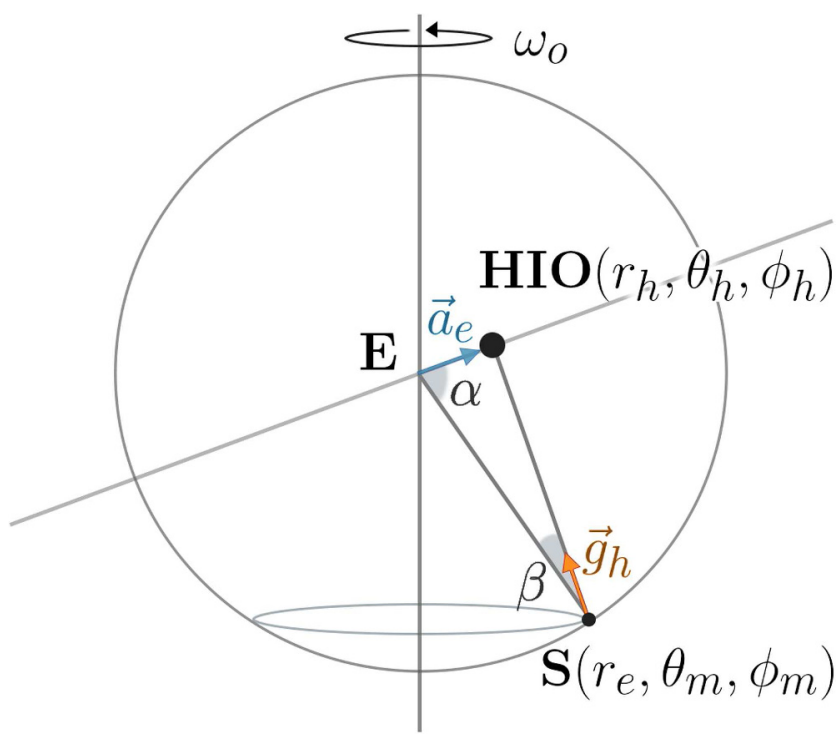

Fig. 2. A diagram of the contribution of the HIO to gravitational acceleration on the Earth's surface with the distance of the HIO to the center exaggerated for clarity. E is the center of the Earth and $\mathrm{S}$ is the location of a gravimeter station. $\vec{a}_{e}$ is the acceleration of the Earth, and $\vec{g}_{h}$ is the gravity provided by the HIO. They both contribute to the overall gravitational acceleration.

In the following derivation we will, for simplicity, assume a one-dimensional oscillation rather than the more general elliptical case (a more detailed derivation is presented in Appendix A). In general, we would expect an elliptical orbit (with more circular orbits being favored, as argued above), and the orbital geometry will have a non-negligible impact on the derived spectrum. However, for the sake of simplicity, we take the 1-d case as illustrative. For small one-dimensional oscillations of a HIO near the center of the Earth the oscillation period is

$$
T=\frac{2 \pi}{\omega_{h}}=\frac{2 \pi}{\sqrt{\frac{4 \pi}{3} G \rho_{0}}} \approx 55 \mathrm{~min}
$$

where $\omega_{h}$ is the angular frequency of the oscillation. In such a model, the HIO contributes to the gravitational acceleration on the Earth's surface via two terms: the gravity of the HIO $\left(\vec{g}_{h}\right)$ and the acceleration of the Earth due to the HIO $\vec{a}_{e}$; see Figure 2. Since the components transverse to the main acceleration of the Earth's gravity would only have second-order corrections to the readings of a scalar gravimeter, the overall effect of a HIO is reduced to:

$$
\delta g \approx g_{h} \cos \beta+a_{e} \cos \alpha .
$$

Note that for small oscillations $\cos \beta \approx 1$, which we take to hold from here on.

Let us introduce an Earth-centered coordinate system with its $z$ axis pointing to the North Pole and denote the direction of an oscillating $\mathrm{HIO}$ as $\left(\theta_{h}, \phi_{h}\right)$. Suppose a gravimeter is placed at $\left(\theta_{m}, \phi_{m}\right)$ on the surface. The relationship between $\alpha$ and the Earth-centered coordinate 
system is:

$\cos \alpha=\sin \theta_{h} \sin \theta_{m} \cos \left(\omega_{0} t+\phi_{m}-\phi_{h}\right)+\cos \theta_{h} \cos \theta_{m}$,

where $\omega_{0}$ is the the angular frequency of the rotation of the Earth. Thus, the gravitational acceleration due to the $\mathrm{HIO}$ would contribute to the gravimeter signal as:

$$
\begin{aligned}
\delta g= & \frac{G m_{h}}{r_{e}^{2}}+\left(2+\frac{\rho_{0}}{\bar{\rho}}\right) \frac{G m_{h}}{r_{e}^{3}} A_{h}\left[\operatorname { s i n } \theta _ { h } \operatorname { s i n } \theta _ { m } \operatorname { c o s } \left(\omega_{0} t\right.\right. \\
& \left.\left.+\phi_{m}-\phi_{h}\right)+\cos \theta_{h} \cos \theta_{m}\right] \cos \omega_{h} t
\end{aligned}
$$

where $m_{h}$ and $A_{h}$ are the mass and amplitude of the HIO oscillation, $\bar{\rho}=5.5 \mathrm{~g} \mathrm{~cm}^{-3}$ is the average density of the Earth, and $\rho_{0}=13.1 \mathrm{~g} \mathrm{~cm}^{-3}$ is the density of the Earth's core. In this scenario, the HIO acts as a harmonic oscillator with a specific frequency, and the frequency is split because of the rotation of the Earth. Note however that while this splitting is exactly the sidereal frequency of the Earth's rotation in the simplified case of a 1-d non-corotating oscillation considered here, in general it would depend strongly on the orbital geometry (and for certain orbits be zero, e.g., a circular equatorial orbit). Due to the non-uniformity of the Earth density (see Fig. 1), this spectral pattern holds for oscillations not exceeding $\approx 0.1 r_{e}$ in amplitude. Note that the second term in equation (4) is smaller than the first term by roughly an order of $\gamma=\left(2+\frac{\rho_{0}}{\bar{\rho}}\right) \frac{A_{h}}{r_{e}}$, so assuming that $A_{h} \approx 0.1 r_{e}$, $\gamma \approx 0.4$.

If the amplitude of such oscillation is large, there will be an amplitude-dependent frequency shift and there will appear spectral harmonics of the signal at the third and higher odd harmonics (see Fig. 3). If the orbit is elliptical and has a radius much larger than $0.1 r_{e}$, the motion is generally aperiodic.

In the case of the amplitude of the oscillation being less than $0.1 r_{e}$, any orbit is a linear combination of three orthogonal normal modes. A circular equatorial orbit in the direction of the Earth's rotation, a circular equatorial orbit opposite the Earth's rotation, and a 1-d "orbit" from the north to the south geographic poles. The 1-d polar orbit will have no frequency shift due to the Earth's rotation (as seen by an stationary observer on the Earth's surface), but the other two will have observed frequencies $\omega_{h} \pm \omega_{0}$. Therefore, a general spectrum should be expected to have three peaks at each harmonic, with the spectral triplet centered on $\omega_{h}$ and split by the Earth's rotational frequency. The relative weights of the three peaks will depend on the orbit and indeed if resolvable should uniquely determine it.

Throughout this work, we assume the Earth to be rigid, an approximation that will need to be abandoned in future, more refined analyses. If we assume that the perturbations at the relevant frequency of $0.3 \mathrm{mHz}$ propagate in the Earth with a speed of $6 \mathrm{~km} / \mathrm{s}$ (a reasonable standin for a typical propagation speed of an elastic wave in the Earth), then the time delay is $\approx 15$ min for the signal from a HIO to reach the gravimeter that is most sensitive to it. This is smaller than the $55 \mathrm{~min}$ HIO oscillation period, but the ratio of the two times is only marginally

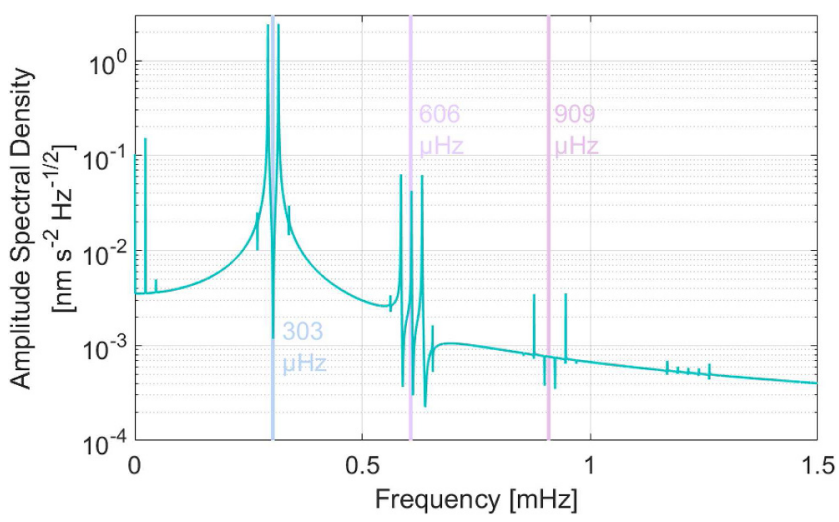

Fig. 3. The theoretical spectrum of a compact HIO undergoing 1-d linear motion near the center of the Earth, on the equatorial plane. The assumed mass of the HIO here is $10^{13} \mathrm{~kg}$, and the amplitude of the oscillation is $0.1 r_{e}$. The amplitude spectral density scales approximately as a product of the HIO mass and the the oscillation amplitude. In the spectrum, there are signals centered near the first (around $303 \mu \mathrm{Hz}$ ) and higher (around $606 \mu \mathrm{Hz}$ and $909 \mu \mathrm{Hz}$ ) harmonics due to the nonlinearity of the force. Rotation of the Earth (seen as a small lowest-frequency peak) also leads to splitting of the first- and second-harmonic lines. Note that the existence and degree of the splitting is not generic, and can vary depending on the particular orbit. The calculation assumes rigid Earth but goes beyond equation (4) by including transverse acceleration and the nonlinearity of the HIO motion. The nonlinearity leads to the second and higher harmonics of the spectrum, while the transverse acceleration additionally leads to the broadening of the spectral lines.

small. In a more refined treatment, one needs to analyze the resonant response of the Earth to determine both the amplitude and phase of the signal. If the signal frequency happens to fall near a resonant deformation mode of the Earth, this may lead to a significant modification of the phase and amplitude of the signal. These aspects can be analysed using the Preliminary Reference Earth Model [2] in terms of the so-called Love numbers.

\section{Global network of superconducting gravimeters}

Superconducting gravimeters (SGs) continuously measure temporal gravity variations with high precision and longterm stability. The observations reflect the integral effects of all periodic and broadband mass variations and deformations induced by a large number of geophysical effects on temporal scales from $1 \mathrm{~s}$ to several years. The long-term operation of the SGs means that all different versions by the producer GWR Instruments Inc. are currently in use, i.e., from the early commercial TT instruments to the latest transportable iGrav SGs [3]. While the characteristics vary between the $\mathrm{SG}$ versions, the ultimate precision is specified by GWR for the observatory SGs as $10^{-3} \mathrm{~nm} / \mathrm{s}^{2}$ resolution in frequency domain with a noise level of better than $1\left(\mathrm{~nm} / \mathrm{s}^{2}\right)^{2} / \mathrm{Hz}$ in the seismic band from 1 to $8 \mathrm{mHz}$ and a long-term stable drift of some $\mathrm{nm} / \mathrm{s}^{2} / \mathrm{a}$. 
SG data sets are available from the database of the International Geodynamics and Earth Tides Service (IGETS) hosted by the Information System and Data Center at GFZ [5]. Originating as Global Geodynamics Project from 1997 to 2015 [18], since 2015 IGETS provides freely accessible data from an increasing number of stations and sensors all over the world (currently 42 and 60 , respectively) to support global geodetic and geophysical studies. The IGETS database provides three levels of data sets from raw gravity and local atmospheric pressure observations sampled at 1 or $2 \mathrm{~s}$ (level 1) to data sets corrected for instrumental perturbations (level 2) to gravity residuals after particular geophysical corrections (level 3 ). Level 3 products are available for 26 stations and 36 sensors processed by EOST Strasbourg at 1 min sampling [19]. These originate from specially processed level 2 products at EOST and are reduced by gravity effects from solid Earth and ocean tides, atmospheric loading, polar motion and length-of-day variations as well as instrumental drift.

The specified SG precision of $10^{-3} \mathrm{~nm} / \mathrm{s}^{2}$ offers great possibilities in combination with stacking methods using gravity data sets from multiple stations of the IGETS network [6]. Theoretically, the sensitivity for the detection of small periodic signals could be enhanced by $\sqrt{\operatorname{lmn}}$ assuming coherent signals with the precision increase $l$ for monthly averages as well as the total number of stations $m$ and months $n$. However, in reality, the instrumental noise from the SGs is not only superseded by station noise [20] but, above all, by a complex and significant uncertainty budget at the $\mathrm{nm} / \mathrm{s}^{2}$ level from the modelling of mass redistributions in the atmosphere, the oceans, and hydrology on a wide range of time scales [21]. In addition, all SG data sets are affected by free oscillations of the Earth excited by large earthquakes in the target frequency range of $0.3 \mathrm{mHz}$ in this study [3].

The gravimeter precision discussed above, assuming that the geophysical effects may eventually be fully subtracted and ignoring other sources of noise, provides grounds for optimistic estimates of what one might hope to ultimately achieve in a search for HIO. For example, with a network with a similar number of stations as the existing one and assuming signals from the stations are added coherently and with on the order of a month of averaging time, the cumulative sensitivity could, in principle, reach on the order of $10^{-7} \mathrm{~nm} / \mathrm{s}^{2}$. Taking an average oscillation amplitude of $A_{h}=0.1 r_{e}(\approx 637 \mathrm{~km})$, the smallest detectable mass of the network would be:

$$
m_{\min }=\left(2+\frac{\rho_{0}}{\bar{\rho}}\right)^{-1} \frac{\delta g r_{e}^{3}}{G A_{h}}=1 \times 10^{8} \mathrm{~kg} .
$$

This mass can be compared to the reference values discussed at the end of Section 2.

\section{Analysis and preliminary results}

The essence of the data processing is as follows. Our analysis technique involves partitioning the time-domain gravity residuals from each station into one-month long

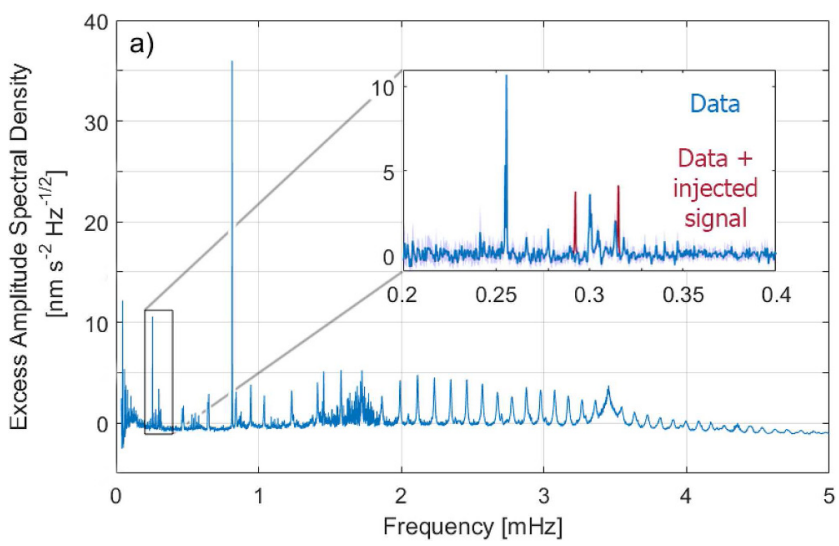

Fig. 4. (a) The amplitude spectral density of the IGETS level 3 data sets, with baseline removal performed after the averaging. The large spike around $800 \mu \mathrm{Hz}$ is due to the ${ }_{0} S_{0}$ "breathing" mode of Earth $[2,22,23]$. The inset (b) shows details around $303 \mu \mathrm{Hz}$, where the signal from a HIO orbiting near the center of the Earth would lie. The dark red line corresponds to the data with the minimum-detectable signal $\left(m=10^{14} \mathrm{~kg}\right)$ injected into it.

segments. We then perform a discrete Fourier transform, normalize by the sampling frequency and the exact segment length, then take the magnitude squared. The resulting quantity is known in the signal-processing literature as a periodogram, which is an estimate of the power spectral density. To improve this estimate, we average all the month-long periodograms from all stations together to produce a single estimate of the true power-spectral density (a process known as Bartlett's method). We take the square root to obtain the amplitude spectral density, then remove any overall baseline by fitting and then subtracting out a function of the form:

$$
y(f)=\frac{A}{f}+\frac{B}{f^{2}}+\frac{C}{f^{3}}+\frac{D}{f^{4}}+y_{0} .
$$

We only fit in the region of higher frequency than tidal effects $(>36.55 \mu \mathrm{Hz})$, to avoid tide-induced fit artifacts. This results in a single averaged spectrum; see Fig. 4a). Some of the spectral features seen in the spectrum are well-known in geophysics. For example, the sharp spike around $800 \mu \mathrm{Hz}$ is the fundamental radial mode ${ }_{0} \mathrm{~S}_{0}[22]$. Other modes in the region of interest have been reported (see e.g., Fig. 21 and the corresponding discussion of the mode identification in Ref. [3]). There are also features in the data that do not generally appear in the raw gravity data from superconducting gravimeters and are possibly artifacts from reduction models used to obtain the level 3 data. This will be subject of further investigation.

We looked for a HIO by fitting three Lorentzians, one centered around $303 \mu \mathrm{Hz}$, and the others centered around $303 \mu \mathrm{Hz} \pm 11.6 \mu \mathrm{Hz}$ (corresponding to a period of 1 day) to the data, and did not detect a signature above noise consistent with the HIO scenario we have presented. To estimate the minimum detectable HIO mass oscillating 
with an amplitude of $0.1 r_{e}$, we injected a signal (from our simulations presented in Fig. 3) into the data. With this, we determined that the minimum injected signal that is detected corresponds to a HIO mass of $\approx 10^{14} \mathrm{~kg}$ at this radius. Details of the analysis can be found in Appendix C. Note that this is not a traditional exclusion limit of a mass, but of a mass on a specific orbit.

This upper bound on the signal, assuming an oscillation $637 \mathrm{~km}\left(\approx 0.1 r_{e}\right)$, would corresponds to an acceleration of $\delta g$ of

$$
\delta g=\left(2+\frac{\rho_{0}}{\bar{\rho}}\right) \frac{m G A_{h}}{r_{e}^{3}}=72 \mathrm{pm} / \mathrm{s}^{2}
$$

Although this upper bound on the mass oscillating with an amplitude of $0.1 r_{e}$ is relatively small $\left(\approx 10^{-10}\right.$ of Earth's mass), the current sensitivity is still several orders of magnitude short of the estimated sensitivity in equation (5). This difference arises from various factors. Although the contributions of the instrument drift and pressure are largely removed already, the employed datafix techniques may introduce errors. Also, there are tides and nontidal loading factors, rainfall and other hydrological factors, station disturbances, seismic factors, and other natural and anthropological contributions to the overall acceleration. Tides generally produce clear, discrete lines in the spectra that are harmonics of the tidal frequency (period $\approx 12 \mathrm{~h}$ ), which have little impact on the region of interest (period $\approx 55 \mathrm{~min}$ ). As for the seismic influences, there are both persistent oscillations, which are a response to other periodic driving forces, which can be roughly evaluated by the Earth model and transient incidences (e.g., earthquakes), which, along with other transient factors, can be removed by data selection. Data selection is also an effective technique for dealing with nontidal and hydrological loading factors which can have an effect of as big as $\approx 1 \times 10^{4} \mathrm{~nm} / \mathrm{s}^{2}$ over a few days. These effects produce noise-like spectra, deteriorating the sensitivity of the network to HIO signals [3].

In the future stages of this work, one should be able to enhance the precision substantially by performing a phase sensitive analysis. Furthermore, the phase information would enable us to approximately determine the specific orbit of a HIO within the Earth, as the phases of a gravimeter signal would depend on its location on Earth's surface.

The stated HIO-mass sensitivity of $10^{8} \mathrm{~kg}$ is based on the assumption that the signals of different stations are completely correlated, and neglects all noise sources other than those of the sensors. In our case where HIO move as a single object inside the Earth, the signal obtained by different stations are indeed correlated. However, if other possible scenarios are to be considered, then the correlation would be incomplete and the sensitivity would deteriorate.

We note that correlation-analysis techniques are currently employed by the existing sensor networks such as LIGO/VIRGO [24] for the detection of gravitational waves, as well as by magnetometer (GNOME [25]) and clock (e.g., GPS.DM [26]) networks for the detection of the galactic dark matter.

\section{Conclusions and outlook}

We have analyzed various possible scenarios of hidden gravitationally bound objects that have weak or no interactions (other than gravitation) with normal matter, and have discussed their possible influence on the total gravitational field measured at the surface of the Earth. With data sets from IGETS, we used Fourier analysis to search for characteristic spectral lines that could be an indication of the existence of such objects. Although no evidence has been found, we estimate that the smallest detectable mass using the current network could perhaps ultimately reach as low as $\approx 1 \times 10^{8} \mathrm{~kg}$. Such hidden gravitationally bound objects could potentially be related to nonbaryonic dark matter.

An alternative scenario to HIO trapped in the Earth is a change of the Earth's gravitational field under the influence of some background bosonic field, for example, that due to dilatons. Serving as dark matter candidates, dilatons and other bosonic fields can have linear interactions with nucleons, changing their effective masses at the Compton frequency associated with the mass. Under appropriate conditions, the mass of the Earth could oscillate slightly at the particle Compton frequency [8]. The superconducting gravimeter data considered in this note are resampled to the once per minute cadence, so the smallest frequency that can be detected is $1 / 120 \mathrm{~Hz}$, corresponding to a particle mass of $3.5 \times 10^{-17} \mathrm{eV}$. The modification of the Earth mass by the presence of an oscillating field $\phi=\phi_{0} \cos \omega_{\phi} t$ is described by

$$
m_{\mathrm{eff}}=\left(1+\frac{\sqrt{\hbar c} \phi}{\Lambda_{1}}\right) m_{e}
$$

where $m_{e}$ is the mass of the Earth, $\omega_{\phi}$ is the frequency of the oscillating field, and $\phi_{0}=\hbar \sqrt{2 \rho_{\mathrm{DM}}} /\left(m_{\phi} c\right)$ is related to the mass of the bosonic particle $m_{\phi}$ and the local density of dark matter $\rho_{\mathrm{DM}} . \Lambda_{1}$ is the coupling constant averaged over all the Earth's atoms.

Assuming the optimistic sensitivity of $10^{-17} \mathrm{~g} \approx$ $10^{-7} \mathrm{~nm} / \mathrm{s}^{2}$ discussed in Section 4 , the network can detect such variance if

$$
m_{\phi} c^{2} \Lambda_{1} \leq 2.5 \times 10^{14} \mathrm{eV}^{2}
$$

which is compatible to the sensitivity of future atom interferometers discussed in reference [8] and performing better than current equivalence-principle tests if $m_{\phi} \leq$ $1 \times 10^{-18} \mathrm{eV}$. Additionally, periodic mass variations of Earth's mass could appear as sidebands in Earth's vibrational modes.

Another possibility is that there could be "boson stars" encountering the Earth that affect the gravitation field. Supposing such influence is only detectable when the distance between the star and the Earth is closer than $10 r_{e}$ and taking the characteristic relative velocity of the Earth and the boson star as the galactic virial velocity $\approx 10^{-3} c$, such transient signal would possibly last $\approx 5 \mathrm{~min}$, which means that its timing is ideal for detection using IGETS's level-2 and level-3 data sets. According to an estimate in [10], the maximum acceleration felt during an 
encounter is $10^{-19} \mathrm{~g}$, so a significant improvement in sensitivity would be needed if one is to detect such events using gravimeters.

In conclusion, advanced gravimeter networks could be useful for detection of exotic matter and future improvements in the hardware and, particularly, in advanced data analysis may enable mounting competitive searches.

Open access funding provided by Projekt DEAL. The authors acknowledge helpful discussions with Joshua Eby, Ernst M. Rasel, Surjeet Rajendran, and the members of the CASPEr and GNOME collaborations. This work was supported in part by the European Research Council (ERC) under the European Union Horizon 2020 research and innovation program (grant agreement No. 695405) and by the DFG via the Reinhart Koselleck project and DFG Project ID 390831469: EXC 2118 (PRISMA + Cluster of Excellence) and by the U.S. National Science Foundation under Grants No. PHY-1707875 and PHY1505903.

\section{Author contribution statement}

W.H. developed the theory of the signals produced by a HIO, performed numerical modelling and wrote parts of the manuscript; M.L. provided a critical assessment of the HIO formation scenarios and their possible connection to dark matter and conducted the analysis of the IGETS data and wrote parts of the manuscript; D.B. coordinated the project and wrote parts of the manuscript; N.L.F. conducted the IGETS data analysis and wrote parts of the manuscript; D.F.J.K. provided theoretical input and wrote parts of the manuscript; A.P.M. proposed the original concept and provided overall scientific oversight; Ch.V. provided input on the geophysical aspects and the operation of the IGETS network and wrote parts of the manuscript. All authors engaged in regular discussions and proofread and edited the paper.

Publisher's Note The EPJ Publishers remain neutral with regard to jurisdictional claims in published maps and institutional affiliations.

Open Access This is an open access article distributed under the terms of the Creative Commons Attribution License (https://creativecommons.org/licenses/by/4.0/), which permits unrestricted use, distribution, and reproduction in any medium, provided the original work is properly cited.

\section{Appendix A: Acceleration at the gravimeter}

In this section, we derive equation (4) step-by-step. As discussed in Section 3, there are two main components for the acceleration measured in a gravimeter station caused by a HIO: one is the direct gravitational attraction of the HIO and another is due to the acceleration of Earth towards the HIO. One would naively expect the acceleration of Earth to be small, but because the HIO is close to the center of the Earth, the effects are of comparable magnitude.
The position vectors of the HIO and the station are described by $\vec{r}_{h}$ and $\vec{r}_{s}$ :

$$
\vec{r}_{h}=\left[\begin{array}{l}
r_{h} \\
\theta_{h} \\
\phi_{h}
\end{array}\right] \quad \vec{r}_{s}=\left[\begin{array}{l}
r_{e} \\
\theta_{s} \\
\phi_{s}
\end{array}\right],
$$

where $\theta$ and $\phi$ denote the respective inclination and azimuthal angles in the spherical coordinate system centered at Earth (see Fig. 2). The gravitational force that pulls Earth towards the HIO is given by,

$$
\vec{F}=\underbrace{\frac{\vec{r}_{h}}{r_{h}}}_{\hat{r}_{h}} \frac{G m_{h}}{r_{h}^{2}} \underbrace{\left(\frac{4 \pi}{3} r_{h}^{3} \rho_{0}\right)}_{M_{e}\left(r_{h}\right)},
$$

where $\hat{r}_{h}$ is the unit vector pointing towards the HIO and $M_{e}\left(r_{h}\right)$ is the mass enclosed by a sphere of radius $r_{h}$. Then, the acceleration of Earth (assuming its a rigid sphere) towards the HIO is given by:

$$
\left.\vec{a}_{e}=\frac{\vec{F}_{\text {Earth's mass }}^{\frac{4 \pi}{3} r_{e}^{3} \bar{\rho}}}{r_{h}}\right)\left(\frac{G m_{h}}{r_{e}^{3}}\right)\left(\frac{\rho_{0}}{\bar{\rho}}\right) r_{h} .
$$

Note that the sensitive axis of superconducting gravimeter stations point along $\vec{r}_{s}$, and so the contribution of $\vec{a}_{e}$ that will be detected, $\delta g_{1}$, will be given by $\vec{a}_{e} \cdot \vec{r}_{s} / r_{e}=$ $\left|\vec{a}_{e}\right| \cos \alpha$. This can be calculated to be:

$$
\begin{aligned}
\delta g_{1}= & \left(\frac{G m_{h}}{r_{h}^{3}}\right)\left(\frac{\rho_{0}}{\bar{\rho}}\right) r_{h}\left(\sin \theta_{h} \sin \theta_{s} \cos \left(\phi_{s}-\phi_{h}\right)\right. \\
& \left.+\cos \theta_{h} \cos \theta_{s}\right) .
\end{aligned}
$$

Now, let us focus on the acceleration component that stems from direct gravitational interaction between the station and the HIO. Let $\vec{r}_{s h}$ denote the vector that points to the HIO from the station, i.e., $\vec{r}_{s h}=\vec{r}_{h}-\vec{r}_{s}$. The gravitational acceleration at the station due to the HIO will be given by:

$$
\vec{g}_{h}=\hat{r}_{h} \frac{G m_{h}}{r_{s h}^{2}}
$$

As before, this acceleration has to be projected onto the sensitivity axis in order to obtain its effective contribution $\delta g_{2}=\vec{g}_{h} \cdot \vec{r}_{s} / r_{e}=\left|\vec{g}_{h}\right| \cos \beta$. But, since we are considering HIOs near the core of Earth, $\cos \beta \approx 1$, and so, $\delta g_{2} \approx\left|\vec{g}_{h}\right|$. Computing $r_{s h}^{2}$ yields

$$
\begin{aligned}
r_{s h}^{2}= & r_{h}^{2}+r_{e}^{2}-2 r_{h} r_{e}\left(\sin \theta_{h} \sin \theta_{s} \cos \left(\phi_{s}-\phi_{h}\right)\right. \\
& \left.+\cos \theta_{h} \cos \theta_{s}\right),
\end{aligned}
$$

and so,

$\delta g_{2}=$

$\frac{G m_{h}}{r_{h}^{2}+r_{e}^{2}-2 r_{h} r_{e}\left(\sin \theta_{h} \sin \theta_{s} \cos \left(\phi_{s}-\phi_{h}\right)+\cos \theta_{h} \cos \theta_{s}\right)}$, 
which can be simplified by multiplying both numerator and denominator by $\left(r_{e}+2 r_{h}\left(\sin \theta_{h} \sin \theta_{s} \cos \left(\phi_{h}-\phi_{s}\right)+\right.\right.$ $\left.\left.\cos \theta_{h} \cos \theta_{s}\right)\right)$ and neglecting expressions that scale with $r_{h}^{2}$ or higher (remember that since the HIO is near the center of Earth, so $r_{h}$ is smaller than $r_{e}$ ), resulting in

$$
\begin{aligned}
\delta g_{2}= & \frac{G m_{h}}{r_{e}^{2}}+\frac{2 G m_{h}}{r_{e}^{3}} r_{h}\left(\sin \theta_{h} \sin \theta_{s} \cos \left(\phi_{s}-\phi_{h}\right)\right. \\
& \left.+\cos \theta_{h} \cos \theta_{s}\right) .
\end{aligned}
$$

We now introduce the HIO's oscilation $r_{h}=A_{h} \cos \omega_{h} t$ and the rotation of the station around Earth $\phi_{s}=\phi_{s, 0}+$ $\omega_{0} t$. Finally, the total acceleration is given by $\delta g=\delta g_{1}+$ $\delta g_{2}$, which leads to equation (4):

$$
\begin{aligned}
\delta g= & \frac{G m_{h}}{r_{e}^{2}}+\left(2+\frac{\rho_{0}}{\bar{\rho}}\right) \frac{G m_{h}}{r_{e}^{3}}\left[\operatorname { s i n } \theta _ { h } \operatorname { s i n } \theta _ { s } \operatorname { c o s } \left(\omega_{0} t\right.\right. \\
& \left.\left.+\phi_{s, 0}-\phi_{h}\right)+\cos \theta_{h} \cos \theta_{s}\right] A_{h} \cos \omega_{h} t,
\end{aligned}
$$

as presented in the main text.

\section{Appendix B: Tidal ring-down}

When an object passes through the Earth, its gravity causes the elastic energy of the Earth to change. Part of this energy is dissipated through friction, which could in principle be an indirect method contributing to energy loss of a hidden object.

Consider a point object with mass $M$ crossing the Earth with an initial velocity of $v_{0}$. When the object is at the center of the Earth, the elastic energy of the Earth is maximized. The pressure increment $p(r)$ due to the object inside the Earth can be found from the force balance:

$$
p(r) 4 \pi r^{2}-p(r+d r) 4 \pi(r+d r)^{2}=\frac{4 G M \rho \pi r^{2} d r}{r^{2}} .
$$

Here, the density of the Earth $\rho$, for simplicity of the argument, is assumed to be uniform. Rewriting equation (B.1) as a differential equation with boundary conditions:

$$
-r^{2} \frac{d p}{d r}-2 p r=G M \rho, \quad p\left(r_{e}\right)=0,
$$

we find the solution:

$$
p=\frac{G M \rho\left(r_{e}-r\right)}{r^{2}} .
$$

The deformation of the Earth and the pressure increment can be related via the Young Modulus E:

$$
E \frac{\delta r}{d r}=p
$$

where $\delta r$ is the compression of the layer $d r$. The total elastic energy:

$$
W=\int_{0}^{r_{e}} \frac{1}{2} p 4 \pi r^{2} \delta r=\frac{2 \pi(G M \rho)^{2}}{E} \int_{0}^{r_{e}} \frac{\left(r_{e}-r\right)^{2}}{r^{2}} d r .
$$

This energy diverges at the lower limit of the integral meaning that we must impose some physical cut-off. We can, for instance, assume that the core of the Earth is incompressible and impose a cutoff at $r=r_{c} \approx 1221.5 \mathrm{~km}$, where $r_{c}$ is the core radius (another possible cutoff would come from the finite size of the object $M)$. For $r_{c} \ll r_{e}$, we have:

$$
W=\frac{2 \pi(G M \rho)^{2}}{E} \frac{r_{e}^{2}}{r_{c}} .
$$

The information on the friction inside the Earth can be obtained from seismic data, specifically from the measured $Q$ factors of the seismic oscillations. According to the PREM model [2], a typical $Q$ factor of seismic waves is $Q \approx 10^{3}$. If $M=1 \times 10^{12} \mathrm{~kg}$, the energy loss per pass of an object through the Earth would be roughly

$$
E_{f} \approx \frac{W}{Q} \approx 1 \times 10^{7} \mathrm{~J} .
$$

For comparison, the escape energy of such mass is:

$$
E_{\text {escape }}=\frac{1}{2} M v_{\text {escape }}^{2} \approx 6 \times 10^{19} \mathrm{~J},
$$

so the energy loss is far too small for what is needed to capture the object. Somewhat less pessimistic numbers may be obtained, as can be seen from equation (B.6) for larger masses $M$ and by taking a smaller cut-off radius. Note that Earth's resonances may significantly modify the considerations above.

If the mechanism were to work, an attractive feature is that as the object winds down towards the core, the energy losses decrease because for small oscillations near the Earth center, the energy losses will become quartic in the oscillation amplitude, so the losses effectively turn off, enabling long "ringing" of the oscillation.

\section{Appendix C: Data analysis validation}

To obtain the smallest detectable HIO signal, we inject a synthetic signal into our data set. We vary the amplitude of the injected signal, and find the smallest amplitude we can reliably detect. This amplitude is then converted to a HIO mass in an optimistic orbit (i.e., one with $r=0.1 r_{e}$, and a favorable orbital geometry). In this sense we do not place an exclusion limit on the mass of the HIO, but on a combination of mass and orbital parameters.

The injected signal corresponds to the time-domain acceleration whose spectrum was presented in Figure 3. The signal was scaled by the corresponding mass and injected into the raw time-domain gravity residuals of the SG data sets, with the same phase and amplitude in each injection. The appropriate spectral densities are then computed as normal, by computing and then averaging periodograms. We fit the result to three Lorentzian functions (one at $303 \mu \mathrm{Hz}$, the other two at $303 \pm 11.6 \mu \mathrm{Hz}$. They all have an identical width. This width and the amplitudes are free parameters) using the Nelder-Mead Simplex minimization algorithm and a $\chi^{2}$ cost function. Per-point 

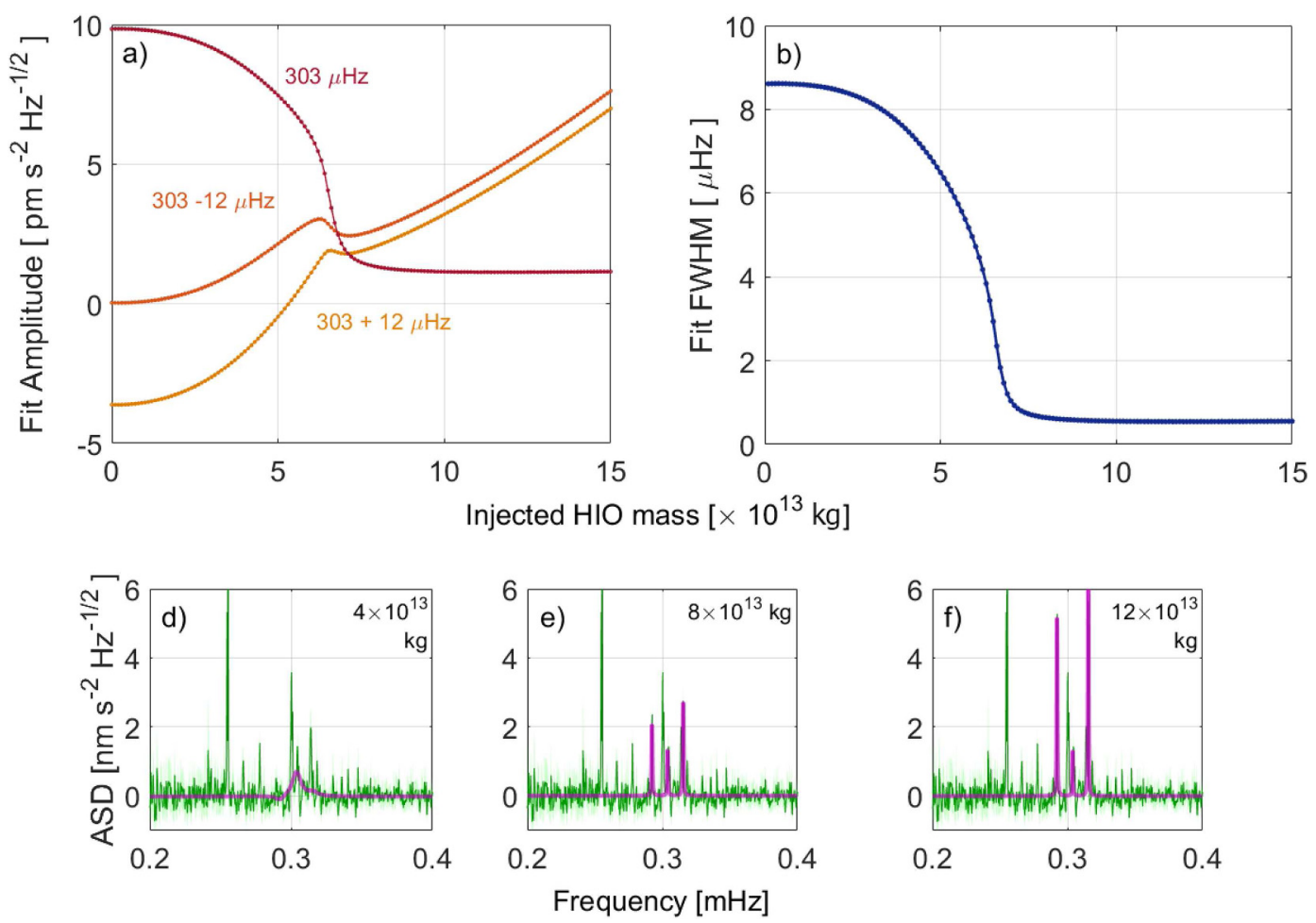

Fig. C.1. Fitting scenarios for different injected amplitudes. (a) and (b) show the behaviour of the fit amplitudes and the width (full width at half maximum (FWHM)) for the three Lorentzians at different injected signals. (c)-(e) are examples of the fit results for injected signals corresponding to a HIO mass of $4 \times 10^{13}, 8 \times 10^{13}$ and $12 \times 10^{13} \mathrm{~kg}$, respectively. The green lines are the data, the light green shaded region represents the standard error of the mean and the purple line corresponds to the fit results.

errors were estimated as the standard deviation of the mean of each frequency point across the periodograms.

The results of the fitting are presented in Figure C.1, the fitting algorithm locks in noise peaks until an injected mass of about $7 \times 10^{13} \mathrm{~kg}$, where it is able to resolve the injected signal. After that point, the fit amplitude increases linearly with the injected mass as one would expect (see Eq. (4)). The central peak falls to a constant noise value (as for our chosen test orbit, no injected spectral weight is assigned to the central peak). In Figure C.1b, we see that the FWHM of the fitted function falls until the algorithm finds the true injected peak, and then becomes constant as expected.

We therefore set a limit at a combination of HIO mass and orbital dynamics resulting in from a HIO mass of $10^{14} \mathrm{~kg}$, corresponding to a gravitational signal strength of $\approx 72 \mathrm{pm} / \mathrm{s}^{2}$. We note that given that this sensitivity is computed using a specific injected signal, it could in principle vary depending on the injected orbit. Future analysis should average over spectra produced by a large number of simulated orbital geometries.

\section{References}

1. G. Bertone, D. Hooper, Rev. Mod. Phys. 90, 045002 (2018)

2. A.M. Dziewonski, D.L. Anderson, Phys. Earth Planet. Inter. 25, 297 (1981)
3. J. Hinderer, D. Crossley, R.J. Warburton, 3.04 - superconducting gravimetry, in Treatise on Geophysics, 2nd edn., edited by G. Schubert (Elsevier, Oxford, 2015), pp. $59-115$

4. The web page of international geodynamics and earth tide service, http://igets.u-strasbg.fr/index.php

5. C. Voigt, C. Förste, H. Wziontek, D. Crossley, B. Meurers, V. Pálinkáš, J. Hinderer, J.-P. Boy, J.-P. Barriot, H. Sun, Report on the Data Base of the International Geodynamics and Earth Tide Service (IGETS), Deutsches GeoForschungsZentrum GFZ, 2016

6. S. Rosat, J. Hinderer, D. Crossley, L. Rivera, Phys. Earth Planet. Inter. 140, 183 (2003)

7. H. Ding, B.F. Chao, J. Geophys. Res.: Solid Earth 120, $7261(2015)$

8. A.A. Geraci, A. Derevianko, Phys. Rev. Lett. 117, 261301 (2016)

9. A. Banerjee, D. Budker, J. Eby, H. Kim, G. Perez, Commun. Phys. 3, 1 (2020)

10. D.F.J. Kimball, D. Budker, J. Eby, M. Pospelov, S. Pustelny, T. Scholtes, Y.V. Stadnik, A. Weis, A. Wickenbrock, Phys. Rev. D 97, 043002 (2018)

11. R.L. McNally, T. Zelevinsky, Eur. Phys. J. D 74, 61 (2020)

12. C.-G. Shao, Y.-F. Chen, R. Sun, L.-S. Cao, M.-K. Zhou, Z.-K. Hu, C. Yu, H. Müller, Phys. Rev. D 97, 024019 (2018)

13. C.J. Horowitz, R. Widmer-Schnidrig, Phys. Rev. Lett. 124, 051102 (2020) 
14. K. Lawson, X. Liang, A. Mead, M.S.R. Siddiqui, L. Van Waerbeke, A. Zhitnitsky, Phys. Rev. D 100, 043531 (2019)

15. A.R. Zhitnitsky, J. Cosmol. Astropart. Phys. 2003, 010 (2003)

16. E. Roche, Acad. Sci. Montpellier 1, 1847 (1850)

17. D. Wittman, N. Golovich, W.A. Dawson, Astrophys. J. 869, 104 (2018)

18. D. Crossley, J. Hinderer, G. Casula, O. Frnacis, H.-T. Hsu, Y. Imanishi, G. Jentzsch, J. Kääriänen, J. Merriam, B. Meurers, J. Neumeyer, Eos Trans. Am. Geophys. Union 80, $121(1999)$

19. J.-P. Boy, Description of the level 2 and level 3 IGETS data produced by EOST (2019), https://isdc.gfz-potsdam. de/igets-data-base/documentation/

20. S. Rosat, J. Hinderer, Sci. Rep. 8, 15324 (2018)
21. M. Mikolaj, M. Reich, A. Güntner, J. Geophys. Res.: Solid Earth 124, 2153 (2019)

22. S. Rosat, S. Watada, T. Sato, Earth Planets Space 59, 307 (2007)

23. T.G. Masters, R. Widmer, Free oscillations: frequencies and attenuations, in Global Earth Physics: a handbook of physical constants (1995), Vol. 1, p. 104

24. LIGO Scientific Collaboration, Virgo Collaboration, et al. Class. Quantum Grav. 37, 055002 (2020)

25. S. Afach, D. Budker, G. DeCamp, V. Dumont, Z.D. Grujić, H. Guo, D.F. Jackson Kimball, T.W. Kornack, V. Lebedev, W. Li, H. Masia-Roig, S. Nix, M. Padniuk, C.A. Palm, C. Pankow, A. Penaflor, X. Peng, S. Pustelny, T. Scholtes, J.A. Smiga, J.E. Stalnaker, A. Weis, A. Wickenbrock, D. Wurm, Phys. Dark Univ. 22, 162 (2018)

26. A. Derevianko, Phys. Rev. A 97, 042506 (2018) 